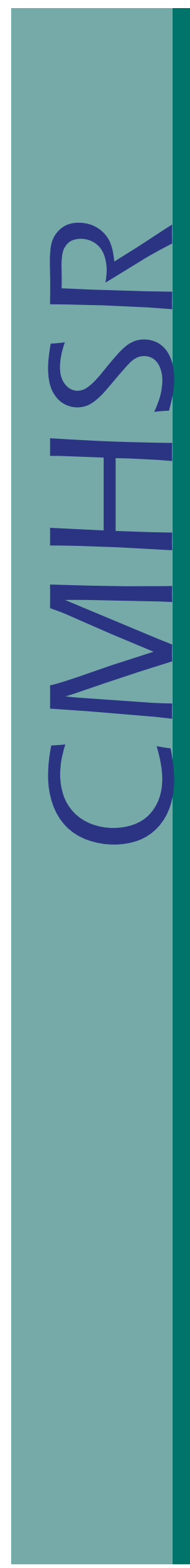

February 2007

Vol 4, Issue 2
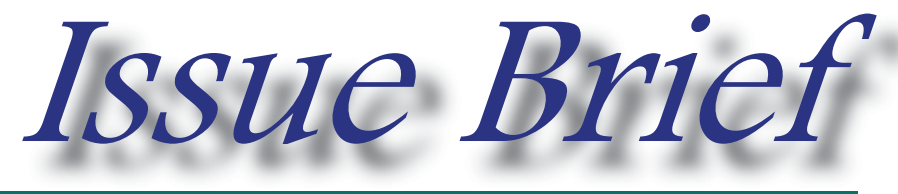

\title{
Maximizing the Results of Internet Surveys
}

Jill Rosenbaum, B.A. \& Charles W. Lidz, Ph.D.

$\mathrm{T}$

Traditionally, researchers have used mail, telephone and face-to-face interview methods to conduct surveys. As the utility of the internet expands, survey researchers are now beginning to capitalize on the popularity of the evolving internet communication method. This shift in technique raises some interesting methodological issues in the realm of survey research. One such issue is the alteration of previously established survey processes to a new methodology. In this brief we describe our adaptation and modification of well-known survey methods to maximize the response rate in a recent internet survey conducted at CMHSR.

\section{Why Choose an Internet Survey?}

Internet surveys have a number of advantages over more traditional survey methods:

Fewer steps for respondents; when using a survey site (we used SurveyMonkey) submission of the survey is online with a link to guide respondents to the survey and a button to submit it. There is no need to mail the survey back or to coordinate a face-to-face interview.

Cost-effective; a yearly membership to SurveyMonkey was $\$ 200$, making it a costeffective alternative to mailing out a survey and providing return postage.

Automated; most survey sites collect and organize the data, as well as track who has taken the survey and who has refused.

(c) 2007 Center for Mental Health Services Research Department of Psychiatry

University of Massachusetts Medical School

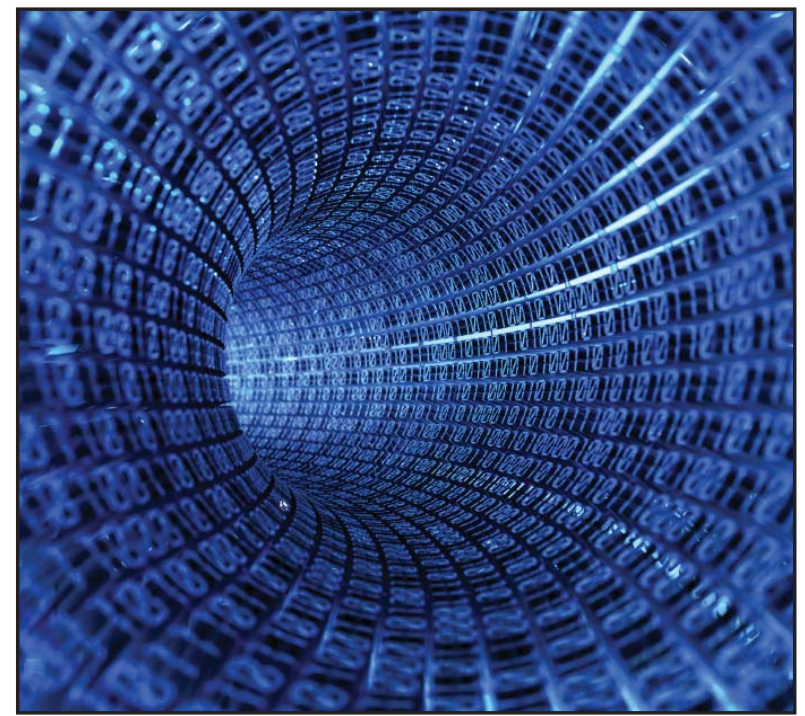

Dillman Method and Modifications

Although internet surveys differ in many ways, there is still a lot to learn from the literature on more traditional survey methods. The Tailored Design Method developed by Don A. Dillman ${ }^{1}$ is widely recognized as the state of the art method for maximizing survey response rates.* Dillman's method is based on extensive research about what improves response rates. Key suggestions from the Tailored Design Method include: making the survey respondent-friendly, including a stamped return envelope, using five varied contacts with survey recipients, providing a financial incentive in the same mailing as the survey itself, and personalizing correspondence. In our work we found it necessary to modify

* It should be noted that in the year since sending out our survey, Dillman ${ }^{2}$ added a section specifically covering internet surveys to his book "Mail and Internet Surveys: The Tailored Design Method." This section was not available when we created our survey but it contains additional information helpful when creating an internet survey. 
Dillman's survey criteria in several ways to suit the internet format. Researchers can create a respondent friendly survey in the usual way, using in-depth interviews with potential participants to guide construction of meaningful survey questions. Researchers should test their questions on a small group of people who fit the study criteria. This step is important for creators of an online survey. The design must be clear and concise because the participants will not have an opportunity to clarify the questions. Since the participants submit the survey online there is no need to include a stamped self-addressed return envelope.

Based on Dillman's suggestion for five varied contacts, we developed a six-point contact method. The initial contact was a letter requesting participation in the study, which looked very similar to letters Dillman suggested. Although Dillman recommended sending a monetary incentive along with the survey, we sent the incentive (a Starbucks Coffee gift card) with the initial contact. Our second contact was simply an email with a link to the survey. The third contact was a postcard thanking those who had participated and reminding those who had not to complete the survey. The next contact was an automated resending of the email with the survey link from the SurveyMonkey address sent only to those who had not responded previously. The fifth contact was a personal telephone call to all non-respondents. Our final contact was an email sent from our study email address (rather than the automated online system) to ensure that recipients' email spam blockers did not prevent our emails from getting through.

We personalized each of these contacts as much as possible. For example, the initial letter was hand signed by study investigators in different colored inks so it was clear that signatures were not printed. Researchers included personal notes requesting current contact information on letters where it seemed likely that the email address was incorrect. Investigators did not use automatic bulk mailing. Instead they adhered individual stamps to all envelopes and postcards. Telephone calls were unscripted so as to follow the sales suggestion that "one needs to sell oneself before one sells the product." We tried to be informal and interested in the respondent.

\section{Limitations of Internet Surveys}

There are some limits to internet surveys. One such limit is that internet surveys constrain the question formats. SurveyMonkey permits 17 different formats and although this is a significant amount, it is not unlimited. Additionally, only certain populations are ideal for internet surveys, limiting the scope with which they can be used. For example, internet surveys may be a poor choice for disadvantaged or impoverished populations as they are unlikely to have access to the required technologies. Also, if the population is likely to have poor reading comprehension, a phone or faceto-face interview might be better options. Thus, it is important to acknowledge that internet surveys can only reach individuals with access to a computer who are moderately computer savvy and capable of comprehending the survey without assistance. However, these limitations are countered by aforementioned advantages, such as ease of respondent participation, cost-effectiveness, automated data collection that can be exported into various software formats, and response tracking.

In summary, we believe that internet surveys, while not perfect tools, have substantial advantages when surveying participants with internet access. The recent survey conducted by the CMHSR achieved an outstanding response rate of $75.4 \%$. This far exceeds the typical response rates on standard mail or phone surveys, which averages $20-30 \%{ }^{1}$

\section{References}

1. Dillman, D.A. (2000). Mail and internet surveys: The Tailored Design Method. New York: John Wiley \& Sons, Inc.

2. Dillman, D.A. (2007). Mail and internet surveys: The Tailored Design Method 2007 update with new internet, visual, and mixed-mode guide. Hoboken, New Jersey: John Wiley \& Sons, Inc.

Visit us on-line at www.umassmed.edu/cmhsr 